OPEN ACCESS

Edited by:

Dimitrios Petrou Bogdanos,

University of Thessaly, Greece

Reviewed by:

Carlo Chizzolini,

Université de Genève, Switzerland

Janine Adele Lamb,

University of Manchester,

United Kingdom

*Correspondence:

Wioleta Marut

w.k.marut@umcutrecht.nl

Specialty section:

This article was submitted to Autoimmune and Autoinflammatory

Disorders,

a section of the journal

Frontiers in Immunology

Received: 11 December 2019

Accepted: 09 April 2020

Published: 22 May 2020

Citation:

Ottria A, Hoekstra AT,

Zimmermann $M$, van der Kroef $M$, Vazirpanah N, Cossu M, Chouri E, Rossato M, Beretta L, Tieland RG,

Wichers CGK, Stigter E, Gulersonmez C, Bonte-Mineur $F$, Berkers CR, Radstake TRDJ and Marut W (2020) Fatty Acid and Carnitine Metabolism Are

Dysregulated in Systemic Sclerosis Patients. Front. Immunol. 11:822. doi: 10.3389/fimmu.2020.00822

\section{Fatty Acid and Carnitine Metabolism Are Dysregulated in Systemic Sclerosis Patients}

\author{
A. Ottria ${ }^{1,2}$, A. T. Hoekstra ${ }^{3}$, M. Zimmermann ${ }^{1,2}$, M. van der Kroef ${ }^{1,2}$, N. Vazirpanah ${ }^{1,2}$, \\ M. Cossu ${ }^{1,2}$, E. Chouri ${ }^{1,2}$, M. Rossato ${ }^{1,2}$, L. Beretta ${ }^{4}$, R. G. Tieland ${ }^{1,2}$, C. G. K. Wichers ${ }^{1,2}$, \\ E. Stigter ${ }^{5}$, C. Gulersonmez ${ }^{5}$, F. Bonte-Mineur ${ }^{6}$, C. R. Berkers ${ }^{3,7}$, T. R. D. J. Radstake ${ }^{1,2}$ \\ and W. Marut ${ }^{1,2 *}$ \\ ${ }^{1}$ Center for Translational Immunology, University Medical Center Utrecht, Utrecht University, Utrecht, Netherlands, \\ ${ }^{2}$ Department of Rheumatology and Clinical Immunology, University Medical Center Utrecht, Utrecht University, Utrecht, \\ Netherlands, ${ }^{3}$ Biomolecular Mass Spectrometry and Proteomics, Bijvoet Center for Biomolecular Research, Utrecht \\ University, Utrecht, Netherlands, ${ }^{4}$ Referral Center for Systemic Autoimmune Diseases, University of Milan and Fondazione \\ IRCCS Ospedale Maggiore Policlinico, Mangiagalli e Regina Elena, Milan, Italy, ${ }^{5}$ Department of Molecular Cancer Research, \\ Center Molecular Medicine, Oncode Institute, University Medical Center Utrecht, Utrecht, Netherlands, ${ }^{6}$ Department of \\ Rheumatology and Clinical Immunology, Maasstad Hospital, Rotterdam, Netherlands, ${ }^{7}$ Department of Biochemistry and Cell \\ Biology, Faculty of Veterinary Medicine, Utrecht University, Utrecht, Netherlands
}

Systemic sclerosis (SSc) is a rare chronic disease of unknown pathogenesis characterized by fibrosis of the skin and internal organs, vascular alteration, and dysregulation of the immune system. In order to better understand the immune system and its perturbations leading to diseases, the study of the mechanisms regulating cellular metabolism has gained a widespread interest. Here, we have assessed the metabolic status of plasma and dendritic cells (DCs) in patients with SSc. We identified a dysregulated metabolomic signature in carnitine in circulation (plasma) and intracellularly in DCs of SSc patients. In addition, we confirmed carnitine alteration in the circulation of SSc patients in three independent plasma measurements from two different cohorts and identified dysregulation of fatty acids. We hypothesized that fatty acid and carnitine alterations contribute to potentiation of inflammation in SSc. Incubation of healthy and SSc dendritic cells with etoposide, a carnitine transporter inhibitor, inhibited the production of pro-inflammatory cytokines such as IL-6 through inhibition of fatty acid oxidation. These findings shed light on the altered metabolic status of the immune system in SSc patients and opens up for potential novel avenues to reduce inflammation.

Keywords: fatty acid oxidation, carnitines, dendritic cells, systemic sclerosis, metabolomics

\section{INTRODUCTION}

Systemic Sclerosis (SSc) is an auto-immune disease with an unknown pathogenesis and unpredictable course. SSc is characterized by vascular lesions, immune cell activation, fibrosis of the skin and internal organs, and loss of the hypodermal fat layer (1). As fat cells are important energy reservoirs, the loss of the fat layer in the fibrotic lesions suggests a role of metabolic changes in SSc. In the last years, metabolomics has shown rapid growth in its application within human health research. The aim of a metabolomics approach is to investigate the complete sets of metabolites within a given sample, in order to achieve a global view of the biological processes within the 
body (2). Many metabolomics studies have already underlined the importance of metabolism in auto-immune diseases and the metabolomics approach has been applied to identify a fingerprint in diseases such as systemic lupus erythematosus (SLE), Sjögren's syndrome (3), multiple sclerosis, and rheumatoid arthritis (2, 46). In SSc, metabolomics pinpoints a distinct metabolic pattern between healthy controls and SSc patients. For instance, a distinct metabolic profile was identified in endothelial cells of SSc patients with pulmonary arterial hypertension (PAH) (7). Other studies have shown a dysregulated fatty acid beta oxidation and amino acid pathway in the urine profile of SSc patients $(2,7-9)$.

Our group has investigated the role of dendritic cells (DCs) in the pathogenesis of SSc. Previously, we have observed pathological behaviors of DCs in SSc patients, such as a downregulation of RUNX3 expression (10) or overproduction of pro-inflammatory cytokine CXCL4 in plasmacytoid DCs (pDCs) (11).

It has been shown that activated DCs have a different metabolic profile that supports their pro-inflammatory status (12). In the current study, we investigated whether metabolomics assessments in the circulation and intracellularly in DCs of SSc patients, could reveal any metabolic aberrances that might contribute to the pathophysiology of SSc. Therefore, we explored the metabolic profile of the plasma of a large cohort of SSc patients followed by a translational experimental setup. Our results indicate changes in the level of carnitine and subsequently fatty acid metabolism to be altered in the circulation and DCs of patients with SSc. Finally, we demonstrated that etoposide, a drug used in cancer therapy, is able to downregulate inflammation in SSc.

\section{METHODS AND PATIENT COHORT \\ Patient Cohort}

In compliance with the guidelines of the Declaration of Helsinki and following the approval of the local Institutional Ethical Review Board, peripheral blood was collected after receiving the written informed consent of the patients. The criteria for selecting patients with SSc was performed according to the 2013 American College of Rheumatology (ACR) (13) Classification. Table 1A represents the characteristics of involved patients from Italian discovery cohort from which the plasma was utilized to perform mass spectrometry assessments. Table $\mathbf{1 B}$ represents the characteristics of involved patients from Dutch validation cohort from which the immune cells and fibroblasts were utilized to perform in vitro assessments. All the blood samples of patients and healthy controls were collected in the morning.

\section{Plasma Collection and Isolation}

Venous blood was collected in a $6 \mathrm{~mL}$ ACD vacutainer (\#364816, $\mathrm{BD}$ Biosciences). Blood was further centrifuged for $10 \mathrm{~min}$ at $1,500 \mathrm{RPM}$ at room temperature in order to obtain plasma. Next, plasma was aliquoted in sterile Micronics tubes and stored in at $-80^{\circ} \mathrm{C}$ freezer until the experiment date.
TABLE 1A | Baseline and clinical characteristics of patients with SSc from the discovery cohort, categorized according to the ACR (2013) criteria (The data are presented as mean \pm SD or min-max).

\begin{tabular}{|c|c|c|c|c|c|}
\hline Discovery cohort & $\begin{array}{c}\mathrm{HC} \\
(N=7)\end{array}$ & $\begin{array}{c}\text { SSc } \\
(N=20)\end{array}$ & $\begin{array}{l}\text { ncSSc } \\
(N=7)\end{array}$ & $\begin{array}{c}\text { IcSSc } \\
(N=6)\end{array}$ & $\begin{array}{l}\text { dcSSc } \\
(N=7)\end{array}$ \\
\hline Age & $59 \pm 14$ & $57 \pm 12$ & $60 \pm 9$ & $59 \pm 10$ & $52 \pm 17$ \\
\hline $\begin{array}{l}\text { Disease duration } \\
\text { (years) }\end{array}$ & - & $13 \pm 11$ & $9 \pm 5$ & $26 \pm 7$ & $7 \pm 7$ \\
\hline Sex (n females) & $7(100 \%)$ & 17 (85\%) & 7 (35\%) & $6(30 \%)$ & $4(20 \%)$ \\
\hline ACR/EULAR score & - & $10 \pm 2$ & $11 \pm 1$ & $12 \pm 2$ & $10 \pm 2$ \\
\hline $\begin{array}{l}\text { Raynaud's } \\
\text { phenomenon (RP) }\end{array}$ & - & 20 (100\%) & 7 & 6 & 7 \\
\hline Puffy fingers (PF) & - & 7 (35\%) & 7 & 0 & 0 \\
\hline Sclerodactyly & - & 12 (60\%) & 0 & 5 & 7 \\
\hline $\begin{array}{l}\text { Digital ulcers (DU) } \\
\text { (anamnestic) }\end{array}$ & - & 5 (25\%) & 1 & 3 & 1 \\
\hline $\begin{array}{l}\text { Modified Rodnan } \\
\text { skin score (mRSS) }\end{array}$ & - & $4(0-27)$ & 0 & $4(0-6)$ & $12(5-27)$ \\
\hline Telangiectasia & - & 10 (50\%) & 1 & 5 & 4 \\
\hline $\begin{array}{l}\text { NVC pattern } \\
\text { (nailfold video } \\
\text { capillaroscopy) }\end{array}$ & - & $9(45 \%)$ & 7 & - & 2 \\
\hline $\begin{array}{l}\text { Anti-nucleus } \\
\text { antibodies (ANA) }\end{array}$ & - & 20 (100\%) & 7 & 6 & 7 \\
\hline $\begin{array}{l}\text { Serum } \\
\text { anticentromere } \\
\text { (ACA) }\end{array}$ & - & 11 (55\%) & 6 & 4 & 6 \\
\hline Autoantibodies & - & $5(25 \%)$ & 0 & 2 & 3 \\
\hline
\end{tabular}

against

topoisomerase I

(scl70)

RVSP (right

ventricular systolic

pressure)

ILD (interstitial lung

disease)

Forced vital

capacity (FVC) (\%

of predicted)

Lung diffusing

capacity for

carbon monoxide

(DLCO) (\% of

predicted)

Nifedipine

Disease-modifying

antirheumatic

drugs (DMARDs)

\section{UNTARGETED ANALYSIS METHODS (ITALIAN DISCOVERY COHORT) \\ Direct-Infusion High Resolution Mass Spectrometry (DIMS)}

Extraction of dried blot spot samples ( $\varnothing 3 \mathrm{~mm}$ ) was performed by ultrasonification for $20 \mathrm{~min}$ in $140 \mu \mathrm{L}$ NSK-AB internal standard solution prepared according to the manufacturer's instructions (Cambridge Isotope Laboratories, Tewksbury, MA, 
TABLE 1B | Baseline and clinical characteristics of patients with SSc from the validation cohort, categorized according to the ACR (2013) criteria.

\begin{tabular}{|c|c|c|c|c|c|}
\hline Validation cohort & $\begin{array}{c}\text { HC } \\
(N=14)\end{array}$ & $\begin{array}{c}S S c \\
\text { validation } \\
(N=12)\end{array}$ & $\begin{array}{l}\text { ncSSc } \\
(N=3)\end{array}$ & $\begin{array}{l}\text { IcSSc } \\
(N=7)\end{array}$ & $\begin{array}{l}\text { dcSSc } \\
(N=2)\end{array}$ \\
\hline Age & $42 \pm 10$ & $53 \pm 9$ & $43 \pm 4$ & $56 \pm 9$ & $55 \pm 3$ \\
\hline $\begin{array}{l}\text { Disease duration } \\
\text { (years) }\end{array}$ & - & $11 \pm 10$ & $8 \pm 7$ & $14 \pm 13$ & $6 \pm 9$ \\
\hline Sex ( $n$ females) & 12 (86\%) & 11 (92\%) & 3 (100\%) & 7 (100\%) & $1(50 \%)$ \\
\hline ACR/EULAR score & - & $12 \pm 2$ & $12 \pm 1$ & $11 \pm 2$ & $14 \pm 2$ \\
\hline $\begin{array}{l}\text { Raynaud's } \\
\text { phenomenon (RP) }\end{array}$ & - & 12 (100\%) & 3 & 7 & 2 \\
\hline Puffy fingers (PF) & - & $7(50 \%)$ & 1 & 3 & 2 \\
\hline Sclerodactyly & - & $5(42 \%)$ & 0 & 3 & 2 \\
\hline $\begin{array}{l}\text { Digital ulcers (DU) } \\
\text { (anamnestic) }\end{array}$ & - & $4(33 \%)$ & 0 & 2 & 2 \\
\hline $\begin{array}{l}\text { Modified Rodnan } \\
\text { skin score (mRSS) }\end{array}$ & - & $7(0-19)$ & 0 & $8(4-10)$ & $16(14-19)$ \\
\hline Telangiectasia & - & $6(50 \%)$ & 1 & 4 & 1 \\
\hline $\begin{array}{l}\text { NVC pattern } \\
\text { (nailfold video } \\
\text { capillaroscopy) }\end{array}$ & - & $9(75 \%)$ & 1 & 4 & 2 \\
\hline $\begin{array}{l}\text { Anti-nucleus } \\
\text { antibodies (ANA) }\end{array}$ & - & $12(100 \%)$ & 3 & 7 & 2 \\
\hline $\begin{array}{l}\text { Serum } \\
\text { anticentromere } \\
\text { (ACA) }\end{array}$ & - & $3(25 \%)$ & 1 & 2 & 0 \\
\hline $\begin{array}{l}\text { Autoantibodies } \\
\text { against } \\
\text { topoisomerase I } \\
\text { (scl70) }\end{array}$ & - & $6(50 \%)$ & 1 & 4 & 1 \\
\hline $\begin{array}{l}\text { ILD (interstitial lung } \\
\text { disease) }\end{array}$ & - & 2 (16\%) & 0 & 1 & 1 \\
\hline $\begin{array}{l}\text { Disease-modifying } \\
\text { antirheumatic } \\
\text { drugs (DMARDs) }\end{array}$ & - & $7(58 \%)$ & 0 & 5 & 2 \\
\hline
\end{tabular}

ncSSc, non-cutaneous SSc; IcSSc, limited cutaneous SSc; dcSSc, diffuse cutaneous SSc. (The data are presented as mean $\pm S D$ or min-max).

USA). After dilution with $60 \mu \mathrm{L} 0.3 \%$ formic acid, the samples were filtered over a $0.2 \mu \mathrm{m}$ cut-off filter plate (Acroprep, Pall Corporation, Ann Arbor, MI, USA). The samples were collected in a 96-well-plate, sealed to avoid evaporation and subjected to DIMS using an Advion TriVersa NanoMate (Advion, Ithaca, NY, USA) with $5 \mu \mathrm{m}$ ID chip-based infusion and a Q-Exactive Plus mass spectrometer (Thermo Scientific, Bremen, Germany). Mass spectrometry data were acquired in the scan range of $\mathrm{m} / \mathrm{z}$ $70-600$. The system was operated at 140,000 mass resolution in both positive and negative mode $(1.5 \mathrm{~min}$ each at $1.6 \mathrm{kV})$. For high mass accuracy, mass calibration was performed before each experiment and internal lock masses were used (14). Raw data files were converted to mzXML format using MS Convert and processed using an in-house-developed untargeted metabolomics pipeline as well as the HDMB database (accurate mass and isotopic pattern).

\section{Liquid Chromatography Mass Spectrometry (LC-MS)}

A volume of $50 \mu \mathrm{L}$ of the sample was subjected to watermethanol-chloroform extraction (Folch-method 3). After phase separation by centrifugation, both the aqueous and the organic phase containing all lipids were transferred to clean vials and dried under a gentle stream of nitrogen gas at $40^{\circ} \mathrm{C}$.

Prior to analysis, the residue of the aqueous phase was dissolved in $100 \mu \mathrm{L} \mathrm{10 \%}$ acetonitrile in ultrapure water. Analysis was conducted with a Thermo Scientific Acella UHPLC system and an Acquity BEH C-8 column $(1 \times 150 \mathrm{~mm}, 1.7 \mu \mathrm{m})$ kept at $40^{\circ} \mathrm{C}$. The column outlet was coupled to a Thermo Scientific Orbitrap XL equipped with an electrospray ion source using both positive and negative ionization. The mass spectrometer was operated in data directed tandem MS mode. The mobile phases consisted of $6.5 \mathrm{mM}$ ammonium carbonate $\mathrm{pH} 8$ (solvent A) and $6.5 \mathrm{mM}$ ammonium carbonate in methanol (solvent B) in the negative mode. For positive mode analysis, the solvents were $0.1 \%$ formic acid in ultrapure water and $0.1 \%$ formic acid in methanol, respectively. Analysis was started upon injection of $5 \mu \mathrm{L}$ of sample. A 10 min linear gradient of $0-100 \%$ B was started $3 \mathrm{~min}$ after the injection of the sample. The system was kept at $100 \%$ B for the next $4 \mathrm{~min}$, after which the system returned to its starting situation. The total runtime was $22 \mathrm{~min}$ and the flow rate was $150 \mu \mathrm{L}$ per minute (15).

For lipidomic analysis, the residue of the organic phase was dissolved in $100 \mu \mathrm{L} \mathrm{80 \%}$ acetonitrile-20\% isopropanol. Analysis was conducted with the system described above using an Acquity BEH C18 column $(1 \times 100 \mathrm{~mm}, 1.7 \mu \mathrm{m})$ kept at $60^{\circ} \mathrm{C}$. The system was operated at a flow rate of $100 \mu \mathrm{L}$ per minute. The mobile phases consisted of $40 \%$ acetonitrile also containing $10 \mathrm{mM}$ ammonium acetate (solvent A), and 10\% acetonitrile: $90 \%$ isopropanol also containing $10 \mathrm{mM}$ ammonium acetate (solvent B) for both negative and positive mode. A 12 min linear gradient of $40-100 \%$ B was started after the injection of $5 \mu \mathrm{L}$ of the sample. The system was kept at $100 \%$ B for the next 5 min, after which the system returned to its starting situation. The total runtime was 20 min.

For both the analysis of polar and non-polar (lipid) metabolites, the acquired MS-data was processed using MZMine 2 open source software 4 and searched against available databases.

\section{TARGETED ANALYSIS METHODS (DUTCH VALIDATION COHORT)}

\section{Acylcarnitine Analysis}

For each analysis, a volume of $50 \mu \mathrm{L}$ NSK-B internal standard solution was mixed with $50 \mu \mathrm{L}$ of plasma and $300 \mu \mathrm{L}$ acetonitrile, according to the manufacturer's instructions. The sample was centrifuged at $4^{\circ}$ at $14,000 \times \mathrm{g}$ for $5 \mathrm{~min}$, and the supernatant was transferred to a gas chromatography (GC) vial and evaporated to dryness at $40^{\circ} \mathrm{C}$ under a gentle stream of nitrogen. A volume of $100 \mu \mathrm{L}$ freshly prepared butylation reagent was added to the residue. The vial was then vortexed, incubated at $60^{\circ} \mathrm{C}$ for $15 \mathrm{~min}$, and evaporated to dryness at $40^{\circ} \mathrm{C}$ under a gentle stream of nitrogen. The residue was dissolved in $100 \mu \mathrm{L}$ acetonitrile and 
subjected to analysis. Standards and quality control samples were prepared similarly (16).

Samples were analyzed on a Waters XEVO Triple Quadrupole mass spectrometer using an Acquity UPLC system for sample delivery (Waters, Milford, MA USA). For the analysis of the samples, $5 \mu \mathrm{L}$ of the derivatized sample was injected via the systems bypass via a restrictor into an $400 \mu \mathrm{L}$ per minute acetonitrile flow. The MS system was operated in the positive ionization mode using MRM scanning (parent-daughter masses) with analyte dependent collision energy for acetyl-carnitine identification and quantification.

\section{Fatty Acid Analysis}

Arachidonic acid-D8 in methanol $(10 \mu \mathrm{L})$ was added to a sample $(20 \mu \mathrm{L})$ and subjected to water-methanol-chloroform extraction (Folch-method). After phase separation by centrifugation, the organic phase containing all lipids was removed from the vial and dried under a gentle stream of nitrogen gas at $40^{\circ} \mathrm{C}$. The residue was dissolved in chloroform and subjected to clean-up by solid phase extraction (SPE) on amino-silica columns (17). After SPE, the fraction containing the FFA was dried under a stream of nitrogen at $37^{\circ} \mathrm{C}$. The residue was dissolved in $100 \mu \mathrm{L}$ acetonitrile and subjected to LC-MS analysis using an Acella UHPLC coupled to a LTQ-Orbitrap XL MS. The LC was equipped with an Acquity $\mathrm{BEH}-\mathrm{C} 18$ column $(2.1 \times 5 \mathrm{~cm}, 1.7 \mu \mathrm{m})$ and guard column. Fatty acids were separated by means of a 20 min $10-95 \%$ acetonitrile gradient in $0.1 \%$ acetic acid at $300 \mu \mathrm{L}$ per minutes and $60^{\circ} \mathrm{C}$. FFA identification and response quantification was performed using retention time $( \pm 0.1 \mathrm{~min})$ and ion $\mathrm{m} / \mathrm{z}$-values $(\leq 5 \mathrm{ppm})$.

\section{Monocyte-Derived DCs (moDCs) Differentiation and Stimulation}

Peripheral blood mononuclear cells (PBMCs) from HC and SSc patients were isolated by a Ficoll (GE Healthcare) gradient. Monocytes were isolated using an autoMACS Pro Separator (Miltenyi Biotec) according the manufacturer's instructions. Purity was routinely assessed by flow cytometry and above $94 \%$. Monocytes were seeded at a final concentration of 1 million per $\mathrm{ml}$ and cultured in RPMI-GlutaMAX (Thermo Fisher Scientific) for 6 days. Medium was supplemented with 10\% FBS (Biowest), 10,000 I.E. penicillin-streptomycin (Thermo Fisher Scientific), recombinant human granulocyte-macrophage colony-stimulating factor (GM-CSF, $800 \mathrm{IU} / \mathrm{mL}$; R\&D Systems), and recombinant human interleukin-4 (IL-4, $500 \mathrm{IU} / \mathrm{mL}$; R\&D Systems), as previously described (18).

\section{Mass Spectrometry on moDCs}

After 3 and $24 \mathrm{~h}$, moDCs were harvested and centrifuged for $5 \mathrm{~min}$ at $1,000 \mathrm{G}$. Medium samples were collected and the cell pellet was washed with ice-cold PBS. Metabolites were extracted by adding $50 \mu \mathrm{l}$ of ice-cold MS lysis buffer [methanol/acetonitrile/ULC/MS grade water (2:2:1)] to the cell pellet. Samples were shaken for $10 \mathrm{~min}$ at $4^{\circ} \mathrm{C}$ and centrifuged at $14,000 \mathrm{G}$ for $15 \mathrm{~min}$, after which the supernatants were collected for LC-MS analysis. LC-MS analysis was performed on an Exactive mass spectrometer (Thermo Scientific) coupled to a Dionex Ultimate 3000 autosampler and pump (Thermo
Scientific). The MS operated in polarity-switching mode with spray voltages of 4.5 and $-3.5 \mathrm{kV}$. Metabolites were separated using a Sequant ZIC-pHILIC column $(2.1 \times 150 \mathrm{~mm}, 5 \mu \mathrm{m}$, guard column $2.1 \times 20 \mathrm{~mm}, 5 \mu \mathrm{m}$; Merck) using a linear gradient of acetonitrile and eluent A $\left[20 \mathrm{mM}\left(\mathrm{NH}_{4}\right)_{2} \mathrm{CO}_{3}, 0.1 \% \mathrm{NH}_{4} \mathrm{OH}\right.$ in ULC/MS grade water (Biosolve)]. The flow rate was set at 150 $\mu \mathrm{l} / \mathrm{min}$. Metabolites were identified and quantified using Lcquan software (Thermo Scientific) on the basis of exact mass within 10 ppm and further validated by concordance with retention times of standards. Peak intensities were normalized based on total intensities per time point.

\section{Fibroblast Culture and Stimulation}

Dermal fibroblasts obtained from healthy controls and SSc patients were cultured in DMEM medium (Thermo Fisher Scientific) $10 \%$ FBS (Biowest) and 10,000 I.E. penicillinstreptomycin (Thermo Fisher Scientific) at $37^{\circ} \mathrm{C}$ and $5 \% \mathrm{CO}_{2}$. The day prior to the experiment, fibroblasts were seeded at a final concentration of 7,000 cells per $\mathrm{mL}$. Fibroblasts were starved prior to be stimulated with TGF $\beta 2$ (R\&D cat\#302-B2$002 / \mathrm{CF})$. On the day of the experiment the medium was refreshed and supplemented with etoposide at a final concentration of 10 or $1 \mathrm{nM}$.

\section{RT-PCR and Quantitative (q)PCR}

RNA from fibroblasts was isolated using the Allprep Universal miRNA/RNA/DNA kit from Qiagen. Total RNA was reverse transcribed using SuperScript ${ }^{\mathrm{TM}}$ IV kit by Invitrogen. Duplicate PCR reactions were performed using SYBR greenselect Master Mix (Applied Biosciences) and measured with a Quantstudio 12K flex Real-Time PCR detection system. cDNA was amplified using specific primers (RPL13 $\alpha \mathrm{fw}$ : CCTGGAGGAGAAGAGGA AAGAGA rw: TTGAGGACCTCTGTGTATTTGTCAA, TAGLN fw: CTCATGCCATAGGAAGGACC rw: GTCCGAAC CCAGACACAAGT, $\alpha S M A$ fw: CCGACCGAATGCAGAAGGA rw: ACAGAGTATTTGCGCTCCGAA, Colla1 fw: CCAGA AGAACTGGTACATCAGCA rw: CGCCATACTCGAACTG GAAT, CTGF fw: AGCTCGGTATGTCTTCATGCTGGT rw: TTGCGAAGCTGACCTGGAAGAGAA). Relative levels of gene expression were calculated by normalizing to $R P L 13 \alpha$ housekeeping gene. Fold changes (FC) of mRNA were calculated by using the formula $2^{-\Delta \Delta \mathrm{Ct}}$.

\section{Interleukin 6 Quantification Using ELISA}

Interleukin (IL-)6 was quantified in cell-free supernatants using an ELISA-based PeliKine compact ${ }^{\mathrm{TM}}$ human IL-6 kit (Sanquin, Amsterdam, The Netherlands), and this was performed following the manufacturer's instructions.

\section{Viability Assessment on moDCs}

Annexin V-7AAD staining was used to assess cell death. Cells were stained with Annexin V (1:100 dilution, BD Pharmingen) and 7AAD (1:100 dilution, BD Pharmingen) and measured using a FACS Canto Flow Cytometer. The data were further analyzed with BD FACS DIVA software. 


\section{Statistical Analysis}

Statistical analysis was performed via website platformed based pipeline tool: https://www.metaboanalyst.ca. Distance between samples was measured with Pearson. Where appropriate, a Mann-Whitney test or Paired $t$-test was assessed using Graph Pad Prism 8.0 Software. $P$-values smaller than 0.05 were considered as statistically significant.

\section{RESULTS}

\section{Different Metabolic Pattern Between HC and SSc}

After screening the spectra obtained from the plasma of 27 individuals, a total of 157 compounds were identified. Using the online platform from Metaboanalist.ca, we performed $t$-test statistical analyses and a total of 56 compounds were identified as having a different level in plasma from SSc patients compared to HC (Table 2). Next, a heatmap of the 56 identified metabolites was generated and is shown in Figure 1A. Briefly, the heatmap reveals different levels of metabolites involved in processes such as fatty acid oxidation (FAO) [L-carnitine and fatty acid derived esters of carnitine (acyl-carnitines)] or kidney function (such as urea and creatinine) were observed in patients compared to HC. With the same tool, we generated a principal component analysis (PCA) and a Partial Least Squares Discriminant Analysis (PLS-DA) (Supplementary Figure 1, Figure 1). The result of the PCA and PLS-DA revealed clear separation between HC and SSc patients based on the levels of metabolites detected in plasma (Figure 1B). In order to identify the variable most efficient in separating the $\mathrm{HC}$ from the SSc patients, the variable importance in projection (VIP) score was generated. The results are shown in Figure 1C. The VIP score showed that L-carnitine and acylcarnitines were relevant for the distinction between $\mathrm{HC}$ and SSc patients.

\section{SSc Patients Have Dysregulation of Fatty Acids and Carnitines}

Next, we performed the quantitative enrichment analysis script from Metaboanalist.ca pipeline (Figure 2A). We found multiple metabolic processes to be altered in the plasma of SSc patients as compared to HC, involving fatty acid (FA) and L-carnitine, such as mitochondrial beta oxidation of short chain saturated FA, FA metabolism, beta oxidation of very long chain FA, and carnitine synthesis pathways, to be altered in the plasma of SSc patients as compared to HC. These observations, suggested an inter relation of FA and carnitine involvement in SSc patient's metabolic profile.

To confirm our observation, we performed targeted analysis focusing specifically on FA and carnitine. We observed an increase of lauric acid $(P=0.0001)$, myristic acid $(P=0.0009)$, and arachidic acid $(P=0.015)$ (Figure 2B) in the plasma of SSc patients when compared to HC.

Furthermore, we found an increase of the carnitine $(P=$ $0.025)$ and Isovaleryl-carnitine $(P=0.03)$ and a decrease of Octanoyl-carnitine $(P=0.04)$ and Palmitoyl-carnitine $(P=$ 0.06; Figure 2C) in the plasma of SSc patients. These findings are in line with our previous observations using the untargeted panel, further suggesting the presence of an imbalance of FA and carnitines in SSc. To further confirm the alteration of carnitine in the circulation of SSc patients, we measured carnitines using dry blood spot, where we confirmed increased level of carnitines in plasma of SSc patients $(P<0.0001)$. Taken together, we confirmed carnitine alteration in pooled SSc patients using three techniques in independent measurements (Figures 2C, 3A).

\section{Carnitine Alterations in the Immune Cells From SSc Patients}

Furthermore, we investigated whether carnitine alterations were also present at the cellular level in SSc patients. Since the role of dendritic cells in SSc pathogenesis is our main focus, we measured the basal level of carnitine in monocytes derived dendritic cells (moDCs) at two different time points ( 3 and $24 \mathrm{~h}$ ). We observed an increase in L-carnitine after $24 \mathrm{~h}$ incubation $(P=0.023)$ and L-acetyl-carnitine $(P<0.0001$ at $3 \mathrm{~h}$ and $P=0.0086$ at $24 \mathrm{~h})$ in SSc moDCs when compared to HC moDCs (Figure 3B). These results further highlight the potential importance of carnitine in the altered metabolism of SSc patients.

\section{Fatty Acids and Carnitine Levels in SSc Disease Subsets}

In order to gain more insight on FA and carnitine levels per disease subset we performed Mann-Whitney $t$-test per subgroup of $\mathrm{ncSSc}, \mathrm{lcSSc}$, and dcSSc in plasma and dry bloodspots samples. The comparison was made between the subsets and HC (Table 3, Figures 2B,C).

\section{Dysregulation of the Fatty Acid Oxidation and Carnitines in SSc Patients Promotes Inflammation and Fibrosis}

We hypothesize that the alteration in FA and carnitines observed in SSc patients are a manifestation of dysregulated FAO. Alternation in FAO leads to increase production of pro-inflammatory cytokines and inflammation (19, 20). Inflammation is known to induce fibrosis and therefore, promotes a vicious circle which further endorses the FAO (21) (Figure 4A).

Inflammation and fibrosis are two main features observed in SSc patients. To test the hypothesis that the FAO is dysregulated in SSc, leading to a vicious circle where inflammation and FAO induce each other, we investigated the role of different carnitine inhibitors (etoposide, thioridazine, mildronate, and etomoxir) by testing the production of pro-inflammatory cytokine in immune cells. Etoposide is a molecule with an inhibitory effect on the organic cation/carnitine transporter (OCTN2), while thioridazine inhibits peroxisomal oxidation of lipids $(22,23)$, and mildronate is an inhibitor of the mitochondrial carnitine/acyl-carnitine transporter (24). To study the effect of carnitine inhibitors on the production of pro-inflammatory cytokines, we used SSc and HC PBMCs. Since PBMCs from SSc patients are known to spontaneously produce pro-inflammatory cytokines, such as interleukin (IL-) 6 (25), we used IL-6 
TABLE 2 | T-test results of the features with different level in plasma from SSc compared to HC.

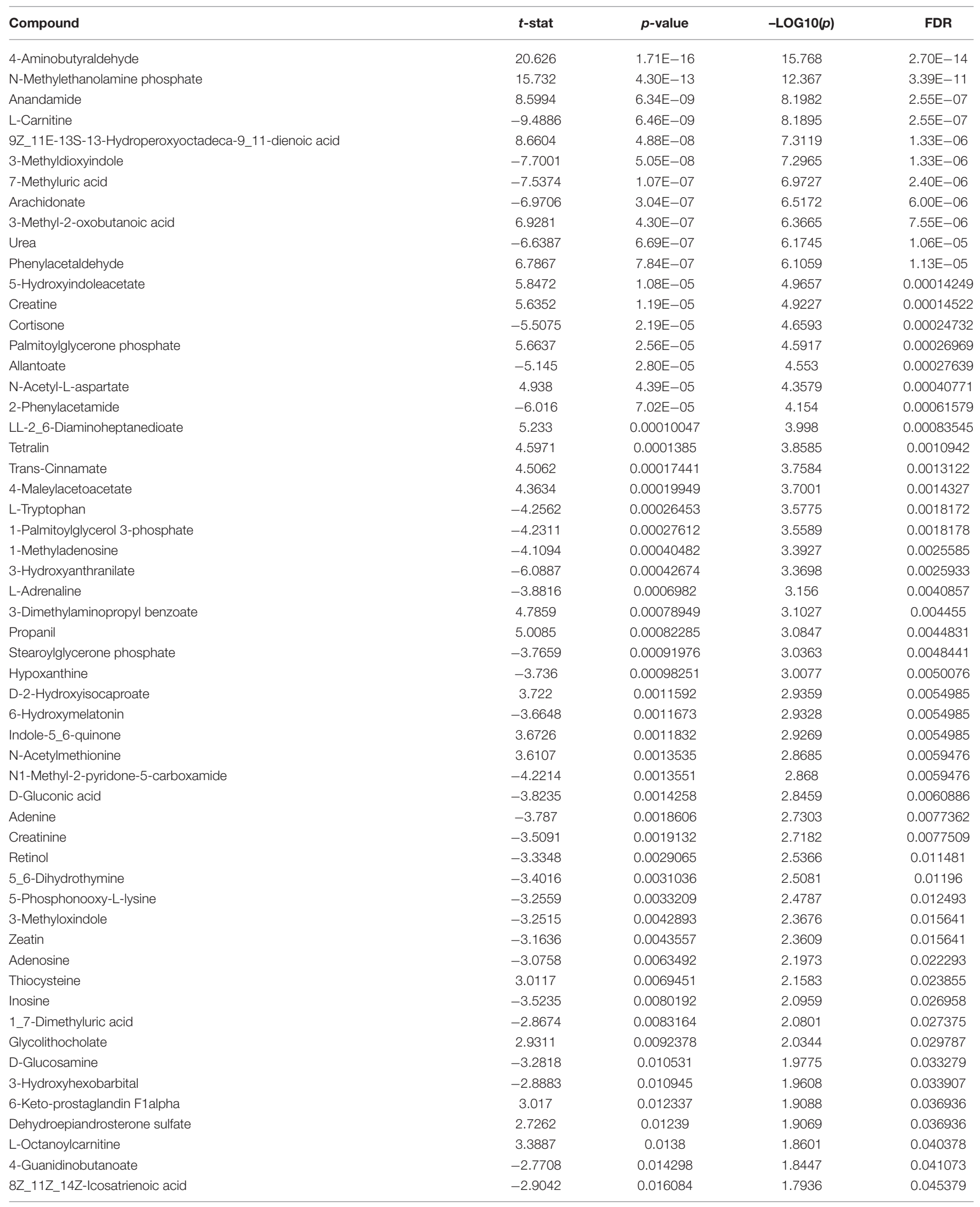

ncSSc, non-cutaneous SSc; ICSSc, limited cutaneous SSc; dcSSc, diffuse cutaneous SSc. 

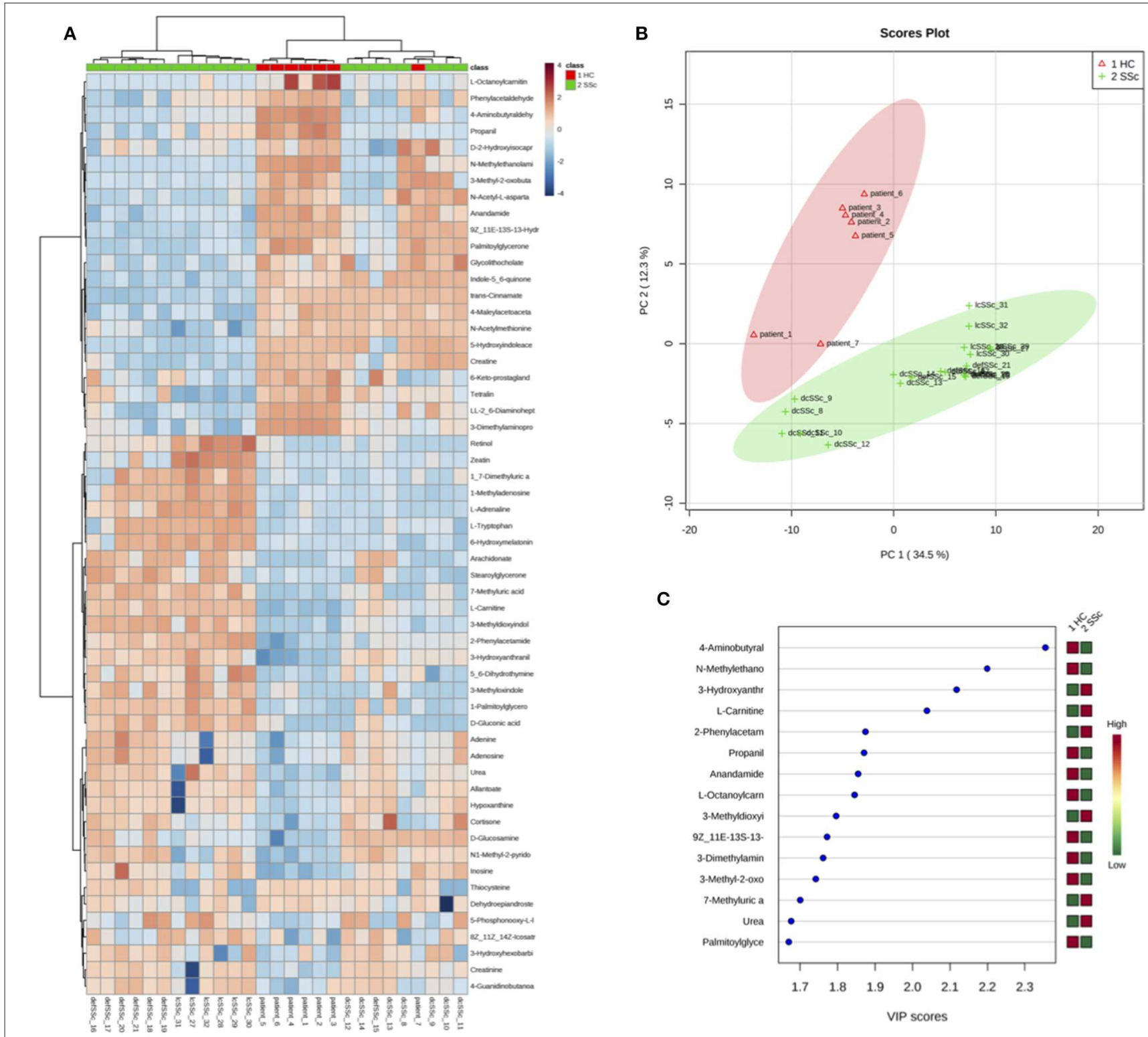

FIGURE 1 | Differentially abundant compounds in plasma from $\mathrm{HC}$ and SSc patients. (A) Heatmap of the 56 significantly different compounds identified in plasma of $\mathrm{HC}$ and SSc patients. (B) Partial Least Squares Discriminant Analysis of plasma samples from HC and SSc patients. (C) Variable Importance in Projection score obtained from the Partial Least Squares Discriminant Analysis.

as readout of the cytokine production. We observed that etoposide $(10 \mathrm{nM})$, etomoxir $(5 \mu \mathrm{M})$ and thioridazine $(10 \mu \mathrm{M})$, but not mildronate $(1 \mathrm{mmol} / \mathrm{L})$, was able to significantly reduce the production of IL-6 $(P=0.0022$ for etoposide and thioridazine and $P=0.0476$ for etomoxir), in PMBCs from SSc patients (Figure 4B). The least variation between the donors were observed with etoposide, and further experiments were therefore performed using etoposide only. Furthermore, etoposide, thioridazine, mildronate, and etomoxir did not affect cell viability (Supplementary Figure 2).

\section{Etoposide Downregulates Inflammatory Response in SSc moDCs and Suppresses Fibrotic Gene Expression in Healthy and SSc Fibroblasts}

Next, moDCs generated from $\mathrm{HC}$ and SSc were stimulated with Poly(I:C) (TLR3 ligand) and cultured in the presence of etoposide for $24 \mathrm{~h}$. We observed no induction of IL-6 in healthy moDCs exposed to TLR3 and/or etoposide. Interestingly, we found a reduction of IL- 6 in SSc moDCs cultured in the presence of etoposide, both, with and without TLR3 stimulation 


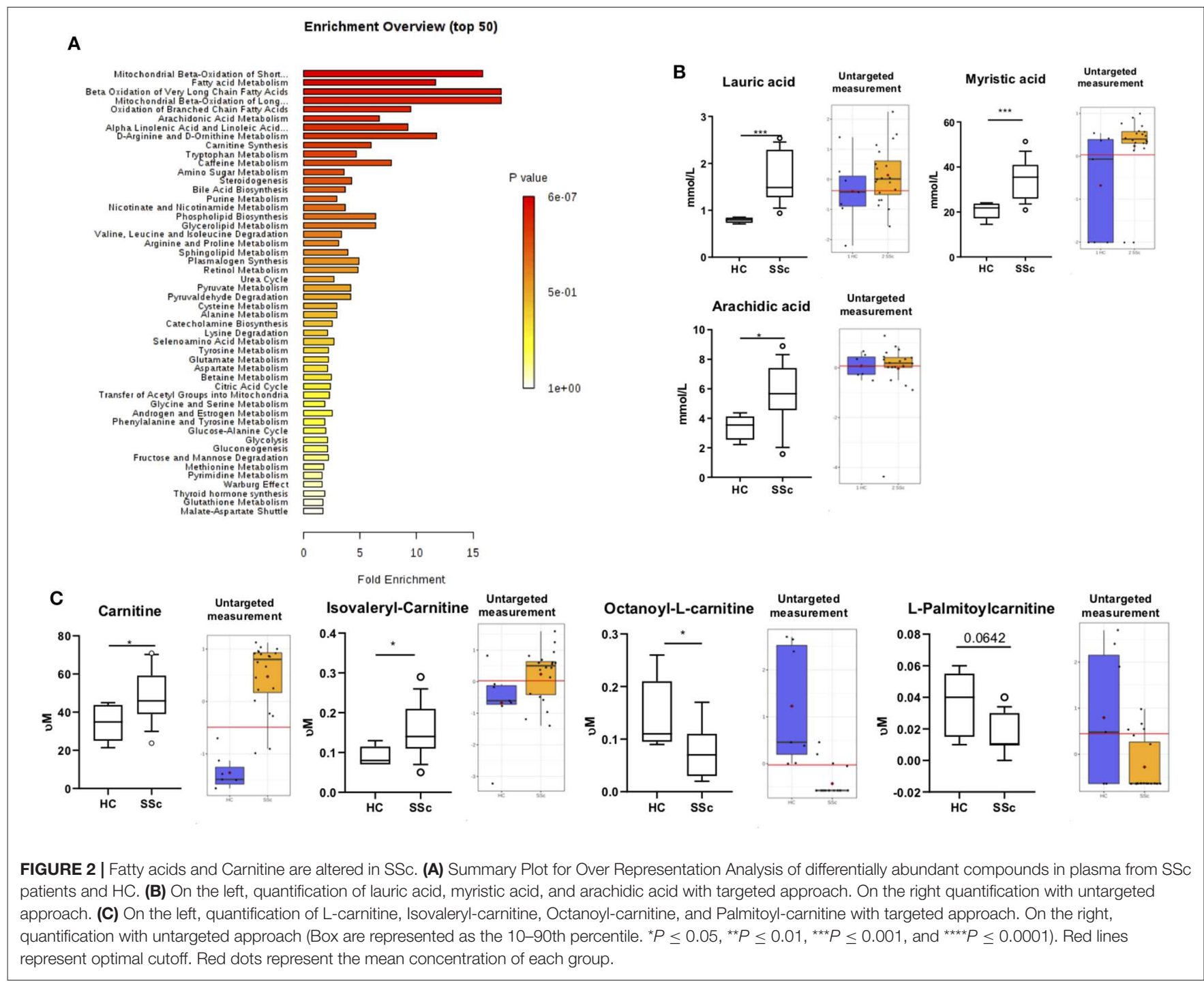

(respectively, $P=0.057$ and 0.028; Figure 4C). Furthermore, we investigated the effect of etoposide on fibrotic genes in healthy fibroblasts stimulated with TGF $\beta 2$ and unstimulated fibroblasts from SSc patients. We measured the expression of transgelin (TAGLN), actin alpha 2 smooth muscle ( $\alpha$ SMA), collagen 1a (Colla) and connective tissue grow factor (CTGF). We observed that, in healthy fibroblasts stimulated with TGF $\beta$, TAGLN $(P=$ $0.029)$, and Colla $(P=0.028)$ were reduced, while $\alpha \mathrm{SMA}(P$ $=0.057)$ and CTGF $(P=0.061)$ showed a trend of reduction (Figure 4D). Unstimulated fibroblasts from SSc patients showed no reduction of TAGLN, $\alpha$ SMA, Colla, and CTGF (Figure 4D). Our data suggests that etoposide have an anti-inflammatory effect on SSc moDCs and an anti-fibrotic effect on healthy fibroblasts stimulated with TGF $\beta$, but not in unstimulated SSc fibroblasts. These results open a new avenue to exploit fatty acid inhibition. Further studies are required in order to delineate the role of etoposide in the mechanism of inflammation involved in SSc given its potential anti-fibrotic effect shown on $\mathrm{HC}$ stimulated fibroblasts.

\section{DISCUSSION}

The aim of our study was to identify and explore the potential role of circulatory and intracellular metabolites in the development of inflammation in SSc patients.

We observed an altered FA and carnitines profile in both the blood and immune cells (plasma and DCs) of SSc patients. Carnitine is a molecule with a structure similar to amino acids that is present in every mammalian species. Carnitine can be both taken up by food or being endogenously produced (26). Carnitine plays an important role in cellular energy metabolism. In fact, carnitine transports fatty acids (as acyl-carnitine) into the mitochondria in order to allow FA to be oxidized (26). Therefore, carnitine and FA, via the FAO, are the fundamental key players for the energy metabolism of cells. It has been shown that an altered FA metabolism is reflected by defective composition of acyl-carnitines (27). In line with this, we observed alteration in acyl-carnitines, such as Isovaleryl-, Palmitoyl-, and Octanoylcarnitine. We speculate that the increase of Isovaleryl-carnitine 


\section{Carnitine \\ A (dry blood spot)}

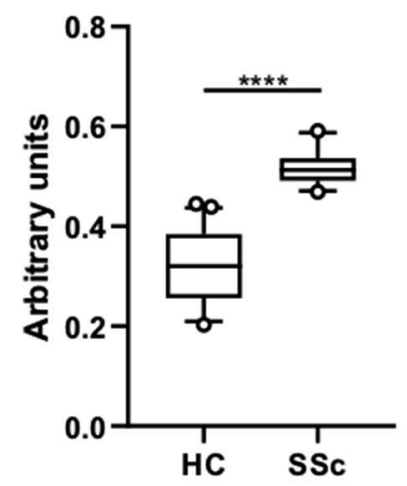

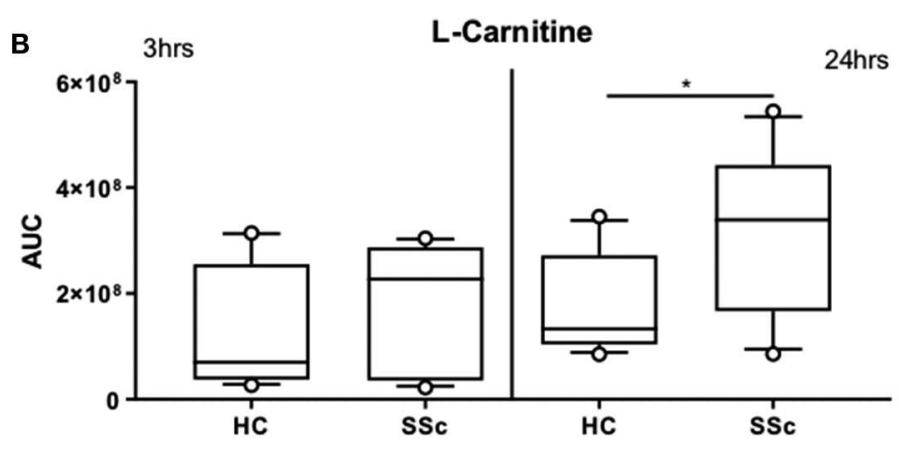

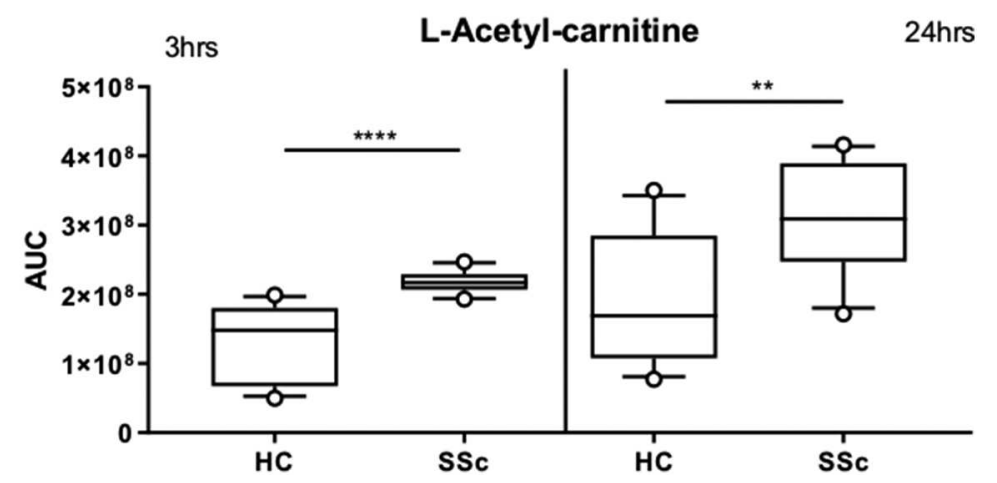

FIGURE 3 | Carnitine is increased in SSc. (A) Quantification of L-carnitine in dry blood spot measurement. (B) Quantification of L-carnitine and L-Acetyl-carnitine in four healthy controls and four SSc moDCs executed in triplicate and incubated for 3 or $24 \mathrm{~h}$ (AUC, Arbitrary unit count; boxes are represented as $10-90 \%$. ${ }^{*} P \leq 0.05$, ${ }^{\star \star} P \leq 0.01,{ }^{* \star *} P \leq 0.001$, and $\left.{ }^{\star \star \star \star} P \leq 0.0001\right)$.

could derive from an excess of amino acids consumption in order to generate ATP. Furthermore, the reduction observed in Palmitoyl-carnitine levels, a factor known to potentially facilitate the transfer of long-chain FA from cytoplasm into mitochondria during the oxidation of fatty acids $(28,29)$, could imply a dysfunction in the transport of long chain FA inside the mitochondria. This could lead to an imbalance in long chain FA inside of the mitochondria, which could potentially be responsible for the observed low level of Octanoyl-carnitine (a long chain FA derived acyl-carnitine). Taken together, we speculate that our observation of increased Isovaleryl-carnitine could depend on the use by cells of amino acids in order to generate ATP and potentially be induced by a dysfunction in the oxidation of long chain FA.

Briefly, our observations signify an altered energy metabolism in immune cells and plasma of SSc patients that is reflected by a dysregulated FA and carnitine profile, including acyl-carnitines.

Immune cells with altered metabolism lead to aberrant immune response $(30,31)$. In macrophages, increased FA metabolism triggers a disturbed immune response, difficulties in adapting to the surrounding environment, and shifting toward pro-fibrotic M2 phenotype $(32,33)$. Furthermore, studies on $\mathrm{T}$ helper cells showed that an altered FA metabolism and a disturbed (micro)environment surrounding the naïve $\mathrm{T}$ helper cells, predict the metabolic programming of the cells.
TABLE 3 | Statistical comparisons of fatty acid and carnitine levels in plasma and dry blood spots between ncSSc, IcSSc, and dcSSc vs. healthy controls.

\begin{tabular}{lccc}
\hline Plasma FA & $\begin{array}{c}\text { ncSSc } \\
(\boldsymbol{n}=\mathbf{3})\end{array}$ & $\begin{array}{c}\text { IcSSc } \\
(\boldsymbol{n}=\mathbf{7})\end{array}$ & $\begin{array}{c}\text { dcSS } \\
(\boldsymbol{n}=\mathbf{2})\end{array}$ \\
\hline Lauric-acid & 0.0079 & 0.0043 & 0.016 \\
Myristic-acid & 0.0079 & 0.0043 & 0.11 \\
Arachidic-acid & 0.016 & 0.05 & 0.29 \\
Dry blood spot & $(n=6)$ & $(n=6)$ & $(n=6)$ \\
Carnitine & $<0.0001$ & $<0.0001$ & $<0.0001$ \\
Plasma carnitine & $(n=7)$ & $(n=6)$ & $(n=7)$ \\
Carnitine & 0.2222 & 0.0932 & 0.0159 \\
Isovaleryl-carnitine & 0.553 & 0.0215 & 0.0159 \\
Octanoyl-carnitine & 0.0556 & 0.1111 & 0.2857 \\
L-palmitoyl-carnitine & 0.1429 & 0.0671 & 0.4683 \\
\hline
\end{tabular}

Inhibition of FAO shifts the $\mathrm{T}$ helper cell differentiation more toward the pro-inflammatory $\mathrm{T}$ helper 17 phenotype (34). Therefore, in SSc, an increase of the FA metabolic profile of the immune cells, might worsen the disease prognosis by priming a pro-inflammatory programmed immune system. Moreover, dysfunction of FAO was found to play an important role in the direct induction of renal fibrosis development (35). Both inflammation and FAO amplifies the production 


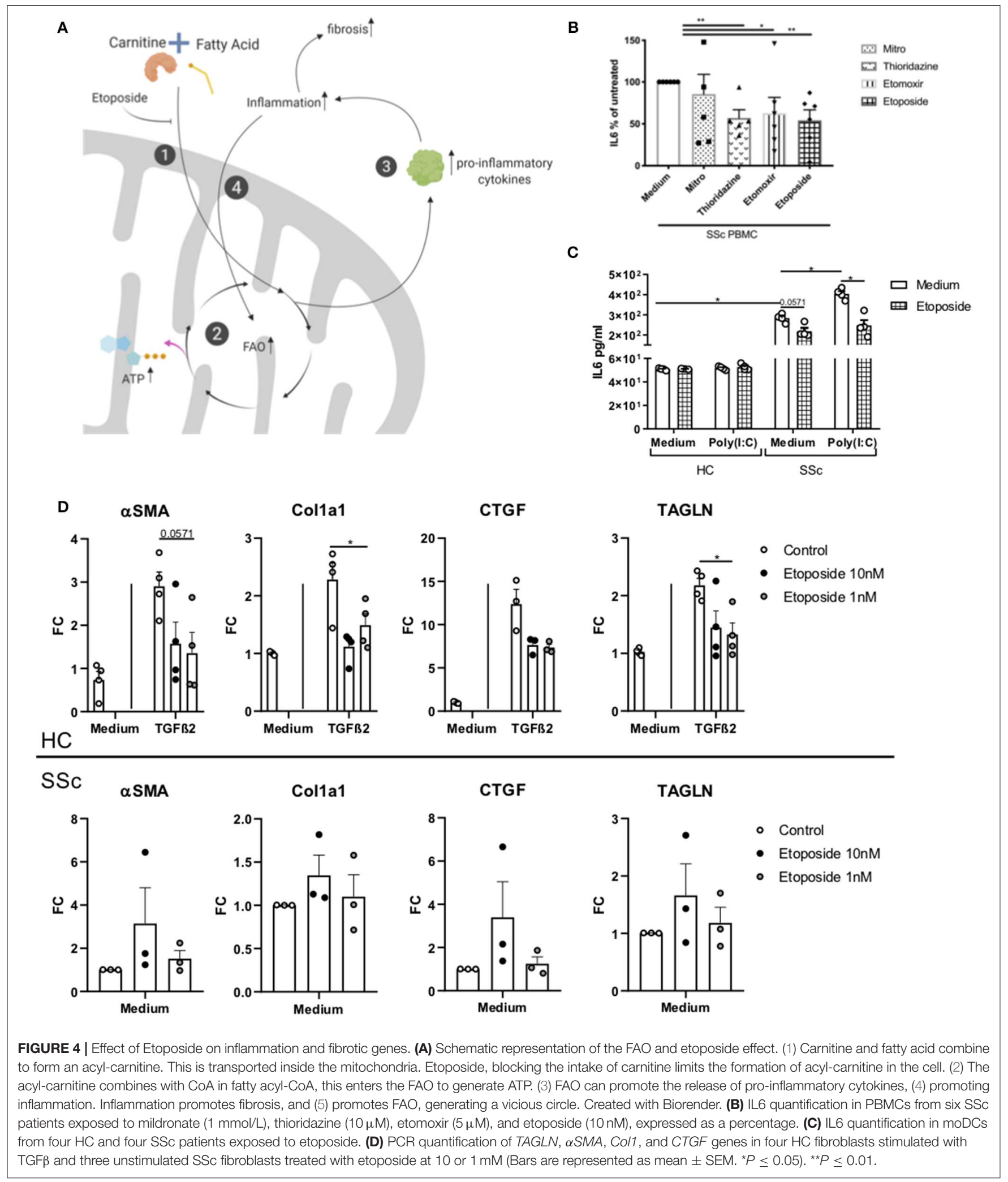

of pro-inflammatory cytokines $(20,21,36)$, and, thereby, they both substantiates the vicious circle of chronic inflammation and fibrosis.
To better understand the significance of FAO in SSc, we blocked the cellular carnitine intake by inhibiting the OCTN2 transporter using etoposide. Etoposide is a well-known drug 
available for cancer treatment as i.e. prostate cancer, small cell lung carcinoma, and leukemia $(37,38)$. Etoposide inhibits carnitine transporter OCTN2 (39), which prevents FAO and subsequently might potentiate the downregulation of proinflammatory immune system. In addition, etoposide has been shown to inhibit topoisomerase II that might impact the experimental results in proliferating cells (40). As monocytederived dendritic cells do not divide in culture and the incubation time with the inhibitor was short, the expected impact on the experimental results is unlikely to occur.

Furthermore, etoposide is suggested to be used in combination with corticosteroids or other DMARDS in treatment of systemic inflammation in Still's disease (41), which is a rare disease with rheumatoid arthritis-like hallmarks. In our studies, etoposide showed anti-inflammatory properties on DCs from SSc and anti-fibrotic properties on TGF- $\beta$ stimulated fibroblasts from healthy donors but not in unstimulated SSc fibroblast. This observation could be explained by potential epigenetic alterations since SSc fibroblast were in vivo chronically exposed to a pro-inflammatory environment. It is indeed known that chronic exposure to stress can induce epigenetic modifications in cells. For instance, cardiac fibroblasts exposed to hypoxia (42) were shown to epigenetically acquire an hyper-reactive fibrotic phenotype. The pro-fibrotic phenotype is acquired after global DNA hypermethylation induced by continuous expression of hypoxia inducible factor $1 \alpha$ (42). Interestingly, hypoxia, together with a dysregulated and chronic immune activation, is the key feature of fibrogenesis in SSc (43). Therefore, it is likely that the overexpression of pro-fibrotic genes in SSc fibroblasts might be eventuated by hypoxia inflicted epigenetic modifications present in patients with SSc. However, further studies need to be performed to support this hypothesis.

One limitation of this study is the absence of information regarding the fasting status of the participants. However, fasting status is not expected to have much influence on the study results since all participants of each cohort (healthy and SSc patients) were included in a similar fashion, and the blood was drawn at approximately the same time.

In conclusion, targeted suppression of the FAO metabolism could be helpful to inhibit inflammation in SSc and therefore might offer a novel therapeutic target. While the literature on FAO and carnitine in SSc is rather poor, we believe that our results provide an intriguing and robust foundation to further elucidate the pathogenic mechanisms taking place in SSc immune dysregulation.

\section{REFERENCES}

1. Pattanaik D, Brown M, Postlethwaite BC, Postlethwaite AE. Pathogenesis of systemic sclerosis. Front Immunol. (2015) 6:272. doi: 10.3389/fimmu.2015.00272

2. Julià A, Vinaixa $M$, Domènech E, Fernández-Nebro $A$, Cañete JD, Ferrándiz C, et al. Urine metabolome profiling of immune-mediated inflammatory diseases. BMC Med. (2016) 14:133. doi: 10.1186/s12916-0160681-8

3. Bellocchi C, Fernández-Ochoa A, Montanelli G, Vigone B, Santaniello A, Quirantes-Piné R, et al. Identification of a shared microbiomic and

\section{DATA AVAILABILITY STATEMENT}

All datasets generated for this study are included in the article/Supplementary Material.

\section{ETHICS STATEMENT}

The studies involving human participants were conducted in compliance with the guidelines of the Declaration of Helsinki and following the approval of the local Institutional Ethical Review Board from UMC Utrecht, Maasstad Ziekenhuis, and Ospedale Maggiore Policlinico, Mangiagalli e Regina Elena. The patients/participants provided their written informed consent to participate in this study.

\section{AUTHOR CONTRIBUTIONS}

All authors approved the final version after being involved in drafting and revising the article for important intellectual content. AO and WM had full access to the data and take responsibility for the accuracy of the performed analysis and the integrity of the data. AO, TR, and WM were involved in design of the study. Execution and analysis of the results was performed by $\mathrm{AO}, \mathrm{AH}, \mathrm{MZ}, \mathrm{MK}, \mathrm{CW}, \mathrm{NV}, \mathrm{MC}, \mathrm{EC}, \mathrm{MR}, \mathrm{LB}, \mathrm{RT}, \mathrm{ES}$, and $\mathrm{CG}$, and they were all involved in performing experiments. AO and $\mathrm{MC}$ were involved in selection of the patients. TR, LB, and FB-M were involved in inclusion of SSc patients. All authors contributed to the review of the manuscript.

\section{FUNDING}

WM obtained funding from Marie Curie Intra-European Fellowship (Proposal number: 624871) and from the NWO VENI (Grant number: 91919149).

\section{ACKNOWLEDGMENTS}

Boudewijn M. T. Burgering helped in revising the paper.

\section{SUPPLEMENTARY MATERIAL}

The Supplementary Material for this article can be found online at: https://www.frontiersin.org/articles/10.3389/fimmu. 2020.00822/full\#supplementary-material

metabolomic profile in systemic autoimmune diseases. J Clin Med. (2019) 8:1291. doi: $10.3390 /$ jcm 8091291

4. Poddighe S, Murgia F, Lorefice L, Liggi S, Cocco E, Marrosu MG, et al. Metabolomic analysis identifies altered metabolic pathways in Multiple Sclerosis. Int J Biochem Cell Biol. (2017) 93:148-55. doi: 10.1016/j.biocel.2017.07.004

5. Wu T, Xie C, Han J, Ye Y, Weiel J, Li Q, et al. Metabolic disturbances associated with systemic lupus erythematosus. PLoS One. (2012) 7:e37210. doi: 10.1371/journal.pone.0037210

6. van Wietmarschen HA, Dai W, van der Kooij AJ, Reijmers TH, Schroën $\mathrm{Y}$, Wang $\mathrm{M}$, et al. Characterization of rheumatoid arthritis subtypes using 
symptom profiles, clinical chemistry and metabolomics measurements. PLoS One. (2012) 7:e44331. doi: 10.1371/journal.pone.0044331

7. Deidda M, Piras C, Cadeddu Dessalvi C, Locci E, Barberini L, Orofino $\mathrm{S}$, et al. Distinctive metabolomic fingerprint in scleroderma patients with pulmonary arterial hypertension. Int J Cardiol. (2017) 241:4016. doi: 10.1016/j.ijcard.2017.04.024

8. Fernández-Ochoa Á, Quirantes-Piné R, Borrás-Linares I, Gemperline D, Alarcón Riquelme ME, Beretta L, et al. Urinary and plasma metabolite differences detected by HPLC-ESI-QTOF-MS in systemic sclerosis patients. J Pharm Biomed Anal. (2019) 162:82-90. doi: 10.1016/j.jpba.2018.09.021

9. Murgia F, Svegliati S, Poddighe S, Lussu M, Manzin A, Spadoni T, et al. Metabolomic profile of systemic sclerosis patients. Sci Rep. (2018) 8:7626. doi: 10.1038/s41598-018-25992-7

10. Affandi AJ, Carvalheiro T, Ottria A, Broen JCA, Bossini-Castillo L, Tieland RG, et al. Low RUNX3 expression alters dendritic cell function in patients with systemic sclerosis and contributes to enhanced fibrosis. Ann Rheum Dis. (2019) 78:1249-59. doi: 10.1136/annrheumdis-2018-214991

11. van Bon L, Affandi AJ, Broen J, Christmann RB, Marijnissen RJ, Stawski L, et al. Proteome-wide analysis and CXCL4 as a biomarker in systemic sclerosis. $N$ Engl J Med. (2014) 370:433-43. doi: 10.1056/NEJMoa1114576

12. O'Neill LAJ, Pearce EJ. Immunometabolism governs dendritic cell and macrophage function. J Exp Med. (2016) 213:1523. doi: 10.1084 /jem. 20151570

13. Van Den Hoogen F, Khanna D, Fransen J, Johnson SR, Baron M, Tyndall A, et al. 2013 classification criteria for systemic sclerosis: an American college of rheumatology/European league against rheumatism collaborative initiative. Arthritis Rheum. (2013) 65:2737-47. doi: 10.1002/art.38098

14. de Sain-van der Velden MGM, van der Ham M, Gerrits J, Prinsen HCMT, Willemsen M, Pras-Raves ML, et al. Quantification of metabolites in dried blood spots by direct infusion high resolution mass spectrometry. Anal Chim Acta. (2017) 979:45-50. doi: 10.1016/j.aca.2017.04.038

15. Rodríguez-Colman MJ, Schewe M, Meerlo M, Stigter E, Gerrits J, Pras-Raves $\mathrm{M}$, et al. Interplay between metabolic identities in the intestinal crypt supports stem cell function. Nature. (2017) 543:424-7. doi: 10.1038/nature21673

16. Pluskal $\mathrm{T}$, Castillo $\mathrm{S}$, Villar-Briones A, Orešič M. MZmine 2: Modular framework for processing, visualizing, and analyzing mass spectrometry-based molecular profile data. BMC Bioinform. (2010) 11:395. doi: 10.1186/1471-2105-11-395

17. Stigter ECA, Letsiou S, vd Broek NJF, Gerrits J, Ishihara K, Voest $\mathrm{EE}$, et al. Development and validation of a quantitative LC-tandem MS assay for hexadeca-4,7,10,13-tetraenoic acid in human and mouse plasma. J Chromatogr B Anal Technol Biomed Life Sci. (2013) 925:169. doi: 10.1016/j.jchromb.2013.01.012

18. Silva-Cardoso SC, Affandi AJ, Spel L, Cossu M, van Roon JAG, Boes M, et al. CXCL4 Exposure potentiates TLR-driven polarization of human monocytederived dendritic cells and increases stimulation of T cells. J Immunol. (2017) 199:253-62. doi: 10.4049/jimmunol.1602020

19. Nicholas DA, Proctor EA, Agrawal M, Belkina AC, Van Nostrand SC, Panneerseelan-Bharath L, et al. Fatty acid metabolites combine with reduced $\beta$ oxidation to activate Th17 inflammation in human type 2 diabetes. Cell Metab. (2019) 30:447-61.e5. doi: 10.1016/j.cmet.2019.07.004

20. Fujieda Y, Manno A, Hayashi Y, Rhodes N, Guo L, Arita M, et al. Inflammation and resolution are associated with upregulation of fatty acid $\beta$-oxidation in zymosan-induced peritonitis. PLoS One. (2013) 8:e66270. doi: 10.1371/journal.pone.0066270

21. Lee SB, Kalluri R. Mechanistic connection between inflammation and fibrosis. Kidney Int. (2010) 78:S22-6. doi: 10.1038/ki.2010.418

22. Shi R, Zhang Y, Shi Y, Shi S, Jiang L. Inhibition of peroxisomal $\beta$-oxidation by thioridazine increases the amount of VLCFAs and $A \beta$ generation in the rat brain. Neurosci Lett. (2012) 528:6-10. doi: 10.1016/j.neulet.2012.08.086

23. Van den Branden C, Roels F. Thioridazine: a selective inhibitor of peroxisomal $\beta$-oxidation in vivo. FEBS Lett. (1985) 187:3313. doi: 10.1016/0014-5793(85)81270-9

24. Oppedisano F, Fanello D, Calvani M, Indiveri C. Interaction of mildronate with the mitochondrial carnitine/acylcarnitine transport protein. J Biochem Mol Toxicol. (2008) 22:8-14. doi: 10.1002/jbt.20208

25. Zhu HL, DU Q, Chen WL, Zuo XX, Li QZ, Liu SJ. Altered serum cytokine expression profile in systemic sclerosis and its regulatory mechanisms. Beijing Da Xue Xue Bao. (2019) 51:716-22. doi: 10.19723/j.issn.1671-167X.2019.04.021

26. Fielding R, Riede L, Lugo JP, Bellamine A. L-carnitine supplementation in recovery after exercise. Nutrients. (2018) 10:349. doi: 10.3390/nu10030349

27. Beger RD, Bhattacharyya S, Gill PS, James LP. Acylcarnitines as translational biomarkers of mitochondrial dysfunction. In: Will Y, Dykens JA, editors. Mitochondrial Dysfunction Caused by Drugs and Environmental Toxicants. Hoboken, NJ: JohnWiley \& Sons, Inc. (2008). p. 383-93.

28. Nałecz KA, Miecz D, Berezowski V, Cecchelli R. Carnitine: transport and physiological functions in the brain. Mol Aspects Med. (2004) 25:55167. doi: 10.1016/j.mam.2004.06.001

29. Goñi FM, Requero MA, Alonso A. Palmitoylcarnitine, a surface-active metabolite. FEBS Lett. (1996) 390:1-5. doi: 10.1016/0014-5793(96)00603-5

30. Loftus RM, Finlay DK. Immunometabolism: cellular metabolism turns immune regulator. J Biol Chem. (2016) 291:110. doi: $10.1074 /$ jbc.R115.693903

31. Pearce EJ, Everts B. Dendritic cell metabolism. Nat Rev Immunol. (2015) 15:18-29. doi: 10.1038/nri3771

32. Freemerman AJ, Johnson AR, Sacks GN, Milner JJ, Kirk EL, Troester MA, et al. Metabolic reprogramming of macrophages: glucose transporter 1 (GLUT1)mediated glucose metabolism drives a proinflammatory phenotype. J Biol Chem. (2014) 289:7884-96. doi: 10.1074/jbc.M113.522037

33. Wang T, Liu H, Lian G, Zhang SY, Wang X, Jiang C. HIF1 $\alpha$-induced glycolysis metabolism is essential to the activation of inflammatory macrophages. Mediators Inflamm. (2017) 2017:1-10. doi: 10.1155/2017/9029327

34. Slack M, Wang T, Wang R. T cell metabolic reprogramming and plasticity. Mol Immunol. (2015) 68:507-12. doi: 10.1016/j.molimm.2015.07.036

35. Allison SJ. Dysfunctional fatty acid oxidation in renal fibrosis. Nat Rev Nephrol. (2015) 11:64. doi: 10.1038/nrneph.2014.244

36. Angajala A, Lim S, Phillips JB, Kim J-H, Yates C, You Z, et al. Diverse roles of mitochondria in immune responses: novel insights into immuno-metabolism. Front Immunol. (2018) 9:1605. doi: 10.3389/fimmu.2018.01605

37. Teicher BA, Silvers T, Selby M, Delosh R, Laudeman J, Ogle C, et al. Small cell lung carcinoma cell line screen of etoposide/carboplatin plus a third agent. Cancer Med. (2017) 6:1952-64. doi: 10.1002/cam4.1131

38. Papiez MA, Krzyściak W, Szade K, Bukowska-Straková K, Kozakowska $M$, Hajduk $K$, et al. Curcumin enhances the cytogenotoxic effect of etoposide in leukemia cells through induction of reactive oxygen species. Drug Des Devel Ther. (2016) 10:557-70. doi: 10.2147/DDDT. S92687

39. Hu C, Lancaster CS, Zuo Z, Hu S, Chen Z, Rubnitz JE, et al. Inhibition of OCTN2-mediated transport of carnitine by etoposide. Mol Cancer Ther. (2012) 11:921-9. doi: 10.1158/1535-7163.MCT-11-0980

40. Baldwin EL, Osheroff N. Etoposide, topoisomerase II and cancer. Curr Med Chem Anticancer Agents. (2005) 5:363-72. doi: 10.2174/1568011054222364

41. Mitrovic S, Fautrel B. Complications of adult-onset Still's disease and their management. Expert Rev Clin Immunol. (2018) 14:351-65. doi: 10.1080/1744666X.2018.1465821

42. Watson CJ, Collier P, Tea I, Neary R, Watson JA, Robinson C, et al. Hypoxiainduced epigenetic modifications are associated with cardiac tissue fibrosis and the development of a myofibroblast-like phenotype. Hum Mol Genet. (2014) 23:2176-88. doi: 10.1093/hmg/ddt614

43. Beyer C, Schett G, Gay S, Distler O, Distler JH. Hypoxia. Hypoxia in the pathogenesis of systemic sclerosis. Arthritis Res Ther. (2009) 11:220. doi: 10.1186/ar2598

Conflict of Interest: The authors declare that the research was conducted in the absence of any commercial or financial relationships that could be construed as a potential conflict of interest.

Copyright (c) 2020 Ottria, Hoekstra, Zimmermann, van der Kroef, Vazirpanah, Cossu, Chouri, Rossato, Beretta, Tieland, Wichers, Stigter, Gulersonmez, BonteMineur, Berkers, Radstake and Marut. This is an open-access article distributed under the terms of the Creative Commons Attribution License (CC BY). The use, distribution or reproduction in other forums is permitted, provided the original author(s) and the copyright owner(s) are credited and that the original publication in this journal is cited, in accordance with accepted academic practice. No use, distribution or reproduction is permitted which does not comply with these terms. 